\title{
Physical Restraint and the Therapeutic Relationship
}

Medium secure psychiatric services in the UK are designed to fulfil a therapeutic function in addition to those of security and custodianship (Mason et al., 2009). The development of strong nurse-patient therapeutic relationships is seen as essential in developing an effective therapeutic milieu in such environments (Thomas, Shattell, \& Martin, 2002). However, in addition to such a therapeutic role, nurses who work in secure settings also have to deal with security issues such as compulsory detention, forced treatment, and risk to others (Mason, Lovell, \& Coyle, 2008). It has been questioned whether staff who work in such services can fulfill both security and therapeutic roles, or whether these roles are incompatible.

Physical restraint activities may be used in a forensic environment as part of routine care, but concerns have been raised about how restraint fits with ethical practice (Mohr, 2010). In addition to being the one who employs the restraint techniques, it is also usually nursing staff who make the decision of whether the patient is restrained. The emotional effect of being involved in restraint or nurses being seen as being part of the system of detention may damage any staff / patient therapeutic alliance (Steele, 1993; . Conacher, 1993).

The literature on the impact of physical restraint on the therapeutic relationship is limited. Research has mainly focused on nursing staff views of physical restraint. For example, Lee, Gray, Gournay, Wright, Parr and Sayer (2003) found that nurses reported that physical restraint was useful in regaining control after an incident, but expressed feelings of ambivalence and concern regarding the after-effects of the restraint. Bigwood and Crowe (2008 found that nurses felt a conflict between their therapeutic role and the milieu of control in the service. They expressed feeling better about physically restraining a patient if they were able to undertake the restraint in a therapeutic way and if they thought that all other options had been explored. Perkins, Prosser, Riley and Whittington (2012) found that nurses 
viewed restraint as a "necessary evil” (p43). Gelkopf, Roffe, Behrbalk et al. (2009) investigated physical restraint in a psychiatric hospital in Israel. Nurses in their sample suggested that the use of restraint showed the patient that staff are unable to 'contain' them otherwise. The nurses reported feeling 'pity', ‘frustration', 'helplessness', ‘appeasement' and 'guilt'. Other research has also suggested that the implementation of restraint may cause strong psychological responses in all parties which then may be present in the therapeutic relationship (Sequeira \& Halstead, 2004). Some participants said that they chose not to display these emotions and instead 'turned them off' so other staff would not see them.

There is a paucity of research which focuses on patient views of restraint and even fewer studies on patients from secure services. Sequeira and Halstead (2002) undertook semistructured interviews with 14 patients in a secure unit to explore their experiences of restraint. The major themes identified included: anger (feelings of injustice and punishment, reenactment of abuse experiences and flashbacks, anger and holding a grudge towards staff), anxiety (scared, panic about the experience and what is going to happen, with some later nightmares reported), mental upset, containment, and release of feelings. Other research has suggested that patients may see restraint as 'being jumped' (Kumar, Guite \& Thornicroft, 2001), or as a form of punishment (Wynn, 2004, Fish \& Culshaw, 2005).

Bonner, Lowe, Rawcliffe and Wellman (2002) undertook a small study focussing on qualitative accounts of both patients and staff of physical restraint. Staff reported that they had seen the restraint as a last resort and highlighted the importance of 'planning, containment and support', whereas patients spoke about strong negative emotions. Importantly, both patients and staff suggested that the strong emotions stayed with them following the restraint. On the basis of this study Bonner et al argued that there was an urgent need for further research on the effects of physical restraint. Wynn (2004) found that patients interviewed around eleven days after being restrained found their hospital experience had become more 
negative following the restraint, reporting the restraint had damaged their relationship with staff and described strong feelings of anger, distrust and fearfulness towards them. It has also been suggested that the restraint of individuals who have experienced trauma such as childhood sexual abuse may re-enact the traumatic experience. For example, Bonner et al. (2002) found that the physical restraint intervention had reminded approximately half of their sample of negative experiences such as childhood abuse and rape, and these patients described being 're-traumatised' by the process. It has been suggested that the process of physical restraint may be a powerful symbolic representation of childhood traumatic experiences (Jennings, 1994). This may be a common experience within mental health inpatient settings due to the high prevalence of previous abuse experiences. One study found that $70.3 \%$ of female patients and $40.6 \%$ of male patients in mental health inpatient settings reported histories of physical or sexual abuse (Shack, Averill, Kopecky, Krajewski, \& Gummattira, 2004).

The literature, although sparse, does suggest that there appears to be a significant role conflict for nurses working in a secure service, and that this might create significant problems in maintaining therapeutic relationships whilst also adopting a security role including the implementation of physical restraint techniques. As there is such, limited research on patient views of this process, our study aimed to explore this issue further through in-depth qualitative interviews with medium secure patients on their perceptions of the impact of restraint on their relationships with staff.

\section{Method}

\section{Setting}

The research was undertaken in a medium secure service in the North of England with low, medium and non-secure beds, and in transitional services for those with longer term 
rehabilitation needs. The service provides assessment, rehabilitation, treatment and long-stay facilities for patients age 18 and above with severe and enduring mental health problems. A high proportion of patients in the service have committed offences which have led them to be detained under the Mental Health Act (1983; 2007) with a restricted Section (requiring Home Office monitoring). The patients detained are thought to present a significant risk to others, in addition to their mental health difficulties.

\section{Participants}

Out of the eight participants interviewed, one was female and seven were male, and participants were aged between 27 and 51 (Mean = 39). All participants described their ethnic group as 'White British'. Participants had been detained in the secure unit between three and 72 months (Mean $=42$ ), and had been in secure care (prisons and secure services) between seven and 336 months $($ Mean $=151)$. The amount of times each participant had been physically restrained ranged from two to 50 times (Mean $=17)$, and the time since last restraint ranged from six weeks to three years (Mean = one year 10 months).

All eight participants were selected using the following inclusion criteria:

- Physically restrained at least once whilst being resident within the current medium secure service

- Detained within the service for at least two months (to ensure that they had the opportunity to engage with, and develop relationships with, staff in the service)

- Able to take part in the interview process

Patients were excluded if the care team felt that participation in the research could have a negative impact on the mental health of the patient, or if there were concerns about the quality and reliability of the data they would provide (e.g. due to current level of mental health difficulties). 


\section{Procedure}

Appropriate ethical approval was gained. Patients who were interested in taking part in the research 'opted in' by completing a slip and placing it in a sealed letter box on the ward area. Of the 16 participants who expressed an interest in taking part, one participant was felt unsuitable for the research due to communication difficulties. A further seven patients were 'opted out' by the care teams because they did meet the inclusion criteria.

Participants were interviewed using a semi-structured interview schedule. The interviews lasted for about 45 minutes and were conducted, recorded and transcribed by the first author.

\section{Analysis}

The data were analysed using thematic analysis, following the guidelines described in Braun and Clarke (2006). The analysis began by reading and gaining familiarity with the data and identifying initial codes (or patterns in the data). Codes were then connected across the transcripts, which were then used to generate themes. These themes were reviewed and refined, and quotes and descriptions are used to illustrate each theme in the results section below.

\section{Findings}

Five main themes were identified in the data, as follows:

\section{Theme 1: Restraint reinforces the inequality of power in the staff-patient} relationship.

Participants acknowledged the underlying power dynamic in the staff-patient relationship. Physical restraint was seen to exacerbate the power and control held by staff; thus creating a greater power imbalance and more of a sense of 'them and us'. Participants 
thought that staff could choose to misuse this power to their advantage. For example, they believed that restraint may be used by some staff in a sadistic way or to manipulate and control patients (theme 4). This led to some patients feeling vulnerable, fearful and distrusting of staff.

Some participants were embarrassed by the power and control that staff held over them. One participant was keen to assure me that this power dynamic would not be present outside of a secure service environment. This suggested that this feeling of powerlessness created by the physical restraint experience was threatening to, and in contrast to, his masculinity and self-image.

Angry. But he were in control of the situation. Because a sad little life, comes here with a bit of power. He wouldn’t be telling me what to do out there (participant 8).

One participant said that they felt infantilised by staff who spoke to them in a disrespectful way. This therefore increased frustration and a feeling of not being understood for the patient.

I just think we're treated like babies, that's it, you know, which is wrong...and they can talk down to you (participant 3).

Participants reported that this power imbalance was highlighted by staff actions throughout the restraint experience. For example, staff used paperwork to document patients' movements ("the power of the pen”) and to justify decisions made. Some participants were untrusting about the need for this documentation, and it was seen as the unknown and a further attempt by staff to control them.

They [staff] can write down anything they want. And you start feeling vulnerable...you start thinking that they can do anything to me here (participant 2). 
In addition to the above, participants reported that staff could make themselves unaccountable by choosing to agree and support each other with regard to decisions made. They explained that all individual staff members, regardless of their personalities and approach, would support each other, sometimes to the detriment of the patient.

You could have the most nicest, friendliest staff member who treats you like a person...you could have someone who is stuck up with rules and policies....But when it comes down to it, they will back each other up on certain things (participant 8).

In terms of staff infantilising patients, this corresponds to the literature focussing on 'paternalistic behaviours' in staff-patient relationships in forensic mental health nursing (Gildberg, Elverdam, \& Hounsgaard, 2010), where patients are seen as people who lack insight and therefore need to be controlled by staff. Controlling interactions may be seen as a punishment by patients, especially as it is opposed to their view of themselves as competent adults (Hinsby \& Baker, 2004).

With regard to staff note taking, previous research by Martin and Street (2003) found that nurses tended to record in patients' case notes in a 'custodial' manner rather than a therapeutic manner. However, they found that semi-structured interviews with the nurses highlighted a more therapeutic approach to nursing.

In any medical environment staff may be seen as the experts and patients as the passive clients who receive care (Welch, 2005). Whilst this is more apparent in terms of physical disease, in terms of psychological distress it may be more intrusive as 'outsiders' become 'experts' on the patient's internal experiences and thoughts. This is further exacerbated in a secure environment, where a powerful security role is also adopted by staff. For staff-patient therapeutic relationships to improve, some of this power needs be shared with the patient (Warne \& McAndrew, 2007), rather than more power being taken away 
through security measures such as physical restraint. This, therefore, creates a significant obstacle to collaborative therapeutic working.

\section{Theme 2: Abusive, degrading, traumatic experience.}

This theme refers to the potentially traumatic impact of physical restraint. Participants suggested that some of the damaging effects of restraint are feeling abused, worthless, helpless and demeaned. Restraint may also trigger post-traumatic stress symptoms. Restraint could also be seen to be a traumatising experience in its own right. The imbalance of power between staff and patients may add to this abusive dynamic where staff are seen as the powerful perpetrators, and the patient as the helpless victim:

Because you are like dirt, and that's what you feel... I was alright five minutes ago before you did this again... it is wrong, it makes you feel helpless (participant 2).

One participant acknowledged the contradiction of staff employing restraint techniques which can have a detrimental impact on patients’ pre-existing mental health difficulties, where the purpose of the patient being detained in the mental health unit was to provide treatment to alleviate these difficulties:

It is degrading being shoved on the floor... Detrimental to your mental health, even though you are in the system where you have got mental health (participant 1).

Participants also labelled restraint as a barbaric, medieval and torturous technique. Most patients blamed staff or the technique itself, during interviews depending on the patient's perception of the staff's motives (theme 4).

One of the participants [participant 5] was distracted throughout the interview. When questioned about this, they stated that they were remembering the physical and psychological pain from one particularly negative restraint experience. They said that they often had vivid 
thoughts and dreams about it, and spent a lot of time preoccupied with these, which suggests that they may have been continuing to react to the traumatic experience of the restraint.

The idea that restraint is a degrading process has also been identified in literature (Gelkopf et al., 2009). However, in this study, the utterances of the participants suggest that the abusive, helpless dynamic of the restraint experience may be linked to the imbalance of power between themselves and the staff . Therefore, restraint can be seen to conflict significantly with the therapeutic milieu that a secure service hopes to provide.

\section{Theme 3: Whether restraint is justified impacts upon whether it is accepted.}

The theme of justification and fairness of the restraint was echoed throughout most interviews, and was thought to be integral to the patient being able to process, understand, and move on from the restraint. Patients could sometimes understand the need for restraint to be employed, and they were able to give examples of when they felt that they had been justifiably restrained. When justified, they suggested that they would take responsibility for their actions and would accept the restraint without feeling anger towards the staff. However, if they felt that staff had restrained them unfairly, they felt hostility and frustration towards the staff, more powerlessness and vulnerability, which would lead to difficulty in future staffpatient interactions This would also lead them to question staff motives

We are all responsible for our own actions....You know if they are in the wrong for doing it...you are not going to be happy are you. If you are in the wrong you admit you are in the wrong and you face whatever you've done (participant 8).

Participants generally thought that restraint was employed too quickly and in situations when it was not required. Participants described a number of situations where they considered restraint had been used unfairly, includingthem raising their voice in an agitated way or when they were completely calm.In the following example, the participant reported 
that they had taken themselves out of the situation which was escalating so that they could use their own personal resources to calm down, and then when they felt calm they re-entered the ward and were immediately restrained.

I had gone in the [room] so I could just relax... Yet they stood outside the [room] and came for me once I had come out.... So there are times where they should restrain, there are times when they shouldn't (participant 8).

Many participants also felt strongly that alternatives (such as staff talking to them to solve the problem or offering different outlets to 'let off steam' had not been explored prior to restraint being used:

They should try and help you before it gets that far...sometimes you go past the anger position don’t you.. (participant 3)

Two participants discussed how when staff perform regular checks of the patient in seclusion, the patient is re-restrained, even if they are feeling calm. . At this point participants said to admit that the incident and restraint was their fault, and asked if they felt any animosity towards staff. One participant reported that if they disagree that it is their fault, or they continue to hold negative feelings towards staff, then they have to lie to be 'let out' of seclusion.

They have you admitting that it was all you. Even if it wasn't you have to say it was, so that then they will decide to let you come out (participant 2).

The findings reflect research undertaken by Duxbury (2002) and Sequeira and Halstead (2002) who found that staff and patients often have differing views about the appropriateness of response by nursing staff to incidents. As highlighted by participants, an important debate around the use of restraint is that of ‘justifiable anger’ (Towl \& Crighton, 1996). This 
concept suggests that anger is a normal emotion which may lead to violence and aggression if the individual has poor coping or anger management skills, or limited ways of expressing their anger.

Lewis (2002) suggested that patients who display aggressive behaviour may be trying to gain and 'reaffirm' their own sense of self, and their right to be angry about their life circumstances. The angry outburst may be an attempt to make sense of their own emotions and experiences (Hopton, 1995). In the interviews, patients asked for their expressions of anger through raising their voices to be allowed, and they requested for other outlets for them to express their emotions and 'let off steam' to be explored before the use of restraint The participants' reported that their understanding of whether the restraint was justified or 'fair' had a significant impact on their experience of the restraint. This was particularly in terms of their feelings towards staff and whether they were able to take responsibility for their own actions and move on, or whether they would feel victimised and unfairly treated by staff leading to feelings of anger and powerlessness. Therefore, in restraints that participants feel are justified, there is a possibility that a more positive staff-patient relationship can be maintained.

\section{Theme 4: The negative attributes and motives of (some) staff.}

Most participants expressed polarised views about staff attributes, describing them as either caring and nice or unemotional beings who liked giving out pain. Most staff were placed in the caring category and were thought to have time for patients, but a few who were said to have a "chip on their shoulder." Some of the negative aspects of the restraint (for example, unjust restraint and the misuse of the power imbalance of the staff-patient relationship) were attributed to ‘negative’ staff. 
Most of the staff here are alright, and we are friendly, but you just get the one who has a got a chip on their shoulder...there is no need for it...We are here to get better, not become worse (participant 3).

Participants proposed the following two possible motives for staff choosing to undertake this type of work which involves physical restraint: either they are doing it for financial reasons (e.g. to pay the mortgage or because it is a good wage), or because they must enjoy the power and inflicting pain on patients. It was also felt that staff must be unemotional and cold to be able to administer an 'abusive' technique (which involves inflicting pain and dominating another human being). Some participants therefore suggested that they themselves would be unable to restrain others, and thus staff working in the service must like giving out pain.

They don't have any feelings towards me about pinning me down....I couldn't do a job like this. I couldn't treat people like this (participant 6).

There was two members of staff on the floor with me... And I believe that they were only doing it to give me pain (participant 5).

A polarised view of staff as either positive or negative has been highlighted as a theme in previous patient research, including a piece by Thomas, Shattell and Martin (2002) who focussed on patient views of the therapeutic milieu of an inpatient unit. Again, this theme identified staff characteristics as important in making this distinction, including whether patients believe that staff are caring.

The suggestion of staff being unemotional may relate to Sequeira \& Halstead's (2004) findings that c staff may attempt to 'turn off' their psychological responses to performing restraint. (). More open communication between patients and staff about the impact of 
physical restraint experiences may be useful in explaining the reactions and behaviours displayed by each party.

\section{Theme 5: Learning to cope with powerlessness during and following restraint.}

The participants described experiencing a feeling of helplessness and powerlessness throughout and following the restraint experience. This theme discusses the diverse strategies patients employ to attempt to cope with these overwhelming feelings.

Many of the participants discussed restraint as a fight, or used fighting terminology when describing restraint. They spoke about their efforts to fight back at staff during the restraint as a way to cope with the powerlessness of this situation, but suggested that they would always 'lose' this fight which led to them feeling helpless and out of control.

Imagine a cagefighter. You get put in them positions...Well, you fight back, but you get mobbed so you can’t do much...They come from everywhere (participant 7).

This attempt at fighting back may also be a way of them expressing some of the emotional pain the restraint may be causing them. However, in some cases, attempts at taking back some control can have negative consequences for the patient, which could lead to greater feelings of hopelessness and vulnerability for the patient.

He [nurse] walked past me and I laughed. And he said what you laughing at, and I said nothing. And he knew I was laughing at him, because that's the only way that I felt that I could stand up for myself...And he turned round and said to me, you seem to be responding to a non-stimuli mate... Now that scared me (participant 2).

Some participants reported that they had learned to suppress negative emotions, and 'play the game' in order to progress through their inpatient stay. Others spoke about their attempts at revenge or attempting to gain some power back over staff. 
I wrote him threatening letters and everything... I memorised his registration plate, he doesn’t work here anymore but I still remember it (participant 8).

Participants also spoke about ways in which they tried to manage their negative emotions in staff-patient interactions. There was a sense amongst participants that their feelings about the restraint experience and associated negative feelings about staff had to be hidden, as if they were aired directly, this could lead to negative consequences. Participants therefore managed these tensions in more subversive ways such as choosing not to engage with staff, or engaging purely in a superficial manner, and making staff aware that they did not like them through non-verbal interactions. This could therefore lead to "uncomfortable silences,” a difficult atmosphere in staff-patient interactions and less openness due to less trust in staff. These difficulties appeared to be solely within interactions with nursing staff involved in the restraint as opposed to all staff. Several participants also spoke about thoughts which were in their mind during interactions with staff following a difficult restraint experience.

But in the back of my mind I'm thinking yeah, you did that... So it's never fully gone (participant 2).

One participant spoke about how he had learned to use these thoughts to his benefit in learning to have some control over his thoughts, and these then not being available to staff.

You just give them a smile and think the most sickest things imaginable. No-one can read your thoughts. It's all we’ve got in here. Everything else gets wrote down (participant 8).

Some literature has highlighted how strong emotions may remain with patients following restraint, including holding a 'grudge’ towards staff (Bonner et al., 2002; Sequeira \& Halstead, 2002). If patients believe they are unable to trust staff members, this would 
clearly have a highly damaging impact upon the staff-patient 'therapeutic' relationship (Berretta et al., 2005). This theme therefore highlights the importance of allowing patients to communicate and address their feelings about the restraint rather than attempting to suppress them, and the importance of de-briefing collaboratively with staff.

\section{Conclusions and Recommendations}

These findings provide an insight into secure service patients’ views of physical restraint and the significant impact of this on the therapeutic relationship with nursing staff. Restraint is seen sometimes as a way for staff to control a difficult, sometimes volatile, environment, but is also sometimes seen as taking advantage of the considerable power imbalance in the staff-patient relationship, which further emphasises this 'gap' between patients and staff. Restraint can also be seen as an abusive experience where patients may experience pain, physical injury, disempowerment and emotional distress, or post-traumatic reactions. In circumstances where restraint is seen as unjust by patients, there are great barriers to subsequently creating or maintaining therapeutic relationships with staff involved. This is exacerbated after the restraint because patients can cope with the difficult feelings of powerlessness and anger or mistrust of staff in which hinder the development of positive relationships with staff.

The findings therefore suggest that in order to facilitate a therapeutic milieu, the ward environment would need to be one which empowers patients to take more control of their own actions and facilitates use of coping strategies and open expression, where staff make use of, and build upon, patients' internal resources and resilience in order to support them. As the therapeutic relationship is based on collaboration, it is extremely important for this power imbalance to be addressed if therapeutic gains are going to be achieved through staff-patient 
interactions. For this reason, regimes that seek to control, and dominate patients in order to manage aggression, are likely to preclude any therapeutic change. .

This leads one to question how physical restraint fits in with the overall model of secure care and the therapeutic milieu which secure services is attempting to provide, as restraint does not appear to be ethically consistent with the therapeutic and recovery model of the service. Consequently, it is essential to develop not only alternatives to restraint, but to ensure that new approaches fit with recovery-oriented practice.

\section{Limitations}

Limitations to this research include not having had the opportunity to build up a relationship or trust with the patients prior to the interview, and therefore, it appeared that some participants had some concerns about talking openly due to a fear of potential consequences. As there was only one female participant, it would be useful if further research could be undertaken to see if there are any gender differences in results.

\section{Clinical Implications}

Many of these clinical implications were generated by the participants within the study and we wish to acknowledge this and reflect upon the validity of their responses. Firstly, it would be beneficial for staff to work with patients to identify ways in which individuals can express their emotions without it resulting in an incident which will lead to restraint (e.g. patients could have the option of using their bedrooms as a 'time out' room from the stressful ward environment). For this to be effective, staff will also need to monitor and be able to recognise signs of patience becoming calmer and regaining their self-control. 
When a patient is in seclusion, staff standing outside of the seclusion room when talking to the patient rather than entering the room and restraining the patient again is likely to help avoid incidents being prolonged.

Questions such as whether patients feel any animosity towards staff following the restraint, or whether they agree that it was 'their fault' should be avoided, but allowing patients to openly express their feelings with regard to the restraint incident without fear of judgement, reprisals or consequences is likely to be beneficial. During the incident questions could usefully focus on what the patient and staff could do to resolve the incident, and whether the patient feels safe to return to the ward area. Implementing such approaches is likely to require specific staff training in many services. Such training might usefully include information about patients' views of physical restraint and how to maintain the therapeutic relationship throughout restraint. Finally, clinical supervision is likely to be a key tool for staff to help them not only to continue to engage in a more 'therapeutic' approach to any use of restraint and seclusion, but also to allow them to discuss the emotional impact on them of undertaking such activities.

\section{References}

Bigwood, S., \& Crowe, M. (2008). 'It's part of the job, but it spoils the job': A phenomenological study of physical restraint. International Journal of Mental Health Nursing, 17, 215-222. doi:10.1111/1447/0349.2008.00526

Bonner, G., Lowe, T., Rawcliffe, D., \& Wellman, N. (2002). Trauma for all: A pilot study of the subjective experience of physical restraint for mental health inpatients and staff in 
the UK. Journal of Psychiatric and Mental Health Nursing, 9, 465-473. doi:10.1046/j.13652850.2002.00504.x

Braun, V., \& Clarke, V. (2006). Using thematic analysis in psychology. Qualitative Research in Psychology, 3, 77-101. doi:10.1191/1478088706qp063oa

Conacher, N. G. (1993). Issues in psychiatric care within a prison service. Canada's Mental Health, 41, 11-15.

Duxbury, J. (2002). An evaluation of staff and patient views of and strategies employed to manage inpatient aggression and violence on one mental health unit: A pluralistic design. Journal of Psychiatric and Mental Health Nursing, 9, 325-337. doi:10.1046/j.1365-2850.2002.00497.x

Fish, R., \& Culshaw, E. (2005). The last resort? Staff and client perspectives on physical intervention. Journal of Intellectual Disabilities, 9, 93-107. doi:10.1177/1744629505049726

Gelkopf, M., Roffe, Z., Behrbalk, P., Melamed, Y., Werbloff, N., \& Bleich, A. (2009). Attitudes, opinions, behaviors, and emotions of the nursing staff toward patient restraint. Issues in Mental Health Nursing, 30, 758-763. doi:10.3109/01612840903159777

Gildberg, F. A., Elverdam, B., \& Hounsgaard, L. (2010). Forensic psychiatric nursing: A literature review and thematic analysis of staff-patient interaction. Journal of Psychiatric and Mental Health Nursing, 17, 359-368. doi:10.1111/j.1365-2850.2009.01533.x.

Hinsby, K., \& Baker, M. (2004). Patient and nurse accounts of violent incidents in a medium secure unit. Journal of Psychiatric and Mental Health Nursing, 11, 341-347. doi:10.1111/j.1365-2850.2004.00736.x. 
Hopton, J. (1995). The contradictions of mental health nursing. Nursing Standard, 8, $37-40$.

Jennings, A. (1994). On being invisible in the Mental Health System. The Journal of Mental Health Administration, 21, 374-387. doi:10.1007/BF02521356

Kumar, S., Guite, H., \& Thornicroft, G. (2001). Patients'experience of violence within a mental health system: A study using grounded theory approach. Journal of Mental Health, 10, 597-611. doi:10.1080/09638230120041353

Lee, S., Gray, R., Gournay, K., Wright, S., Parr, A.-M., \& Sayer, J. (2003). Views of nursing staff on the use of physical restraint. Journal of Psychiatric and Mental Health Nursing, 10, 425-430. doi:10.1046/j.1365-2850.2003.00625.x

Lewis, D. M. (2002). Responding to a violent incident: physical restraint or anger management as therapeutic interventions. Journal of Psychiatric and Mental Health Nursing, 9, 57-63. doi:10.1046/j.1351-0126.2001.00440.x

Martin, T., \& Street, A. F. (2003). Exploring evidence of the therapeutic relationship in forensic psychiatric nursing. Journal of Psychiatric and Mental Health Nursing, 10, 543551. doi:10.1046/j.1365-2850.2003.00656.x

Mason, T., Lovell, A., \& Coyle, D. (2008). Forensic psychiatric nursing: skills and competencies: 1 role dimensions. Journal of Psychiatric and Mental Health Nursing, 15, 118130.

Mason, T., King, L., \& Dulson, J. (2009). Binary construct analysis of forensic psychiatric nursing in the UK: High, medium, and low security services. International Journal of Mental Health Nursing, 18, 216-224. doi:10.1111/j.1447-0349.2009.00601.x 
Mental Capacity Act (2005) UK: HMSO.

Mohr, W. K. (2010). Restraints and the code of ethics: An uneasy fit. Archives of Psychiatric Nursing, 24(1), 3-14. doi:10.1016/j.apnu.2009.03.003.

Perkins, E., Prosser, H., Riley, D., \& Whittington, R. (2012). Physical restraint in a therapeutic setting; a necessary evil? International Journal of Law and Psychiatry, 35, 43-49. doi:10.1016/j.ijlp.2011.11.008.

Sequeira, H., \& Halstead, S. (2002). Control and restraint in the UK: Patient perspectives. The Journal of Forensic Practice, 4, 9-18.

Sequeira, H., \& Halstead, S. (2004). The psychological effects on nursing staff of administering physical restraint in a secure psychiatric hospital: 'When I go home, it's then that I think about it. British Journal of Forensic Practice, 6, 3-15.

Shack, A. V., Averill. P. M., Kopecky, C., Krajewski, K., \& Gummattira, P. (2004). Prior history of physical and sexual abuse among the psychiatric inpatient population: A comparison of males and females. Psychiatric Quarterly, 75, 343-359. doi:10.1023/B:PSAQ.0000043510.41919.55.

Steele, R. L. (1993). Staff attitudes towards seclusion and restraint: Anything new? Perspectives in Psychiatric Care, 29, 23-28.

Thomas, S. P., Shattell, M., \& Martin, T. (2002). What's therapeutic about the therapeutic milieu? Archives of Psychiatric Nursing, 16, 99-107. doi:10.1053/apnu.2002.32945.

Towl, G. J., and Crighton, D. A. (1996). The Handbook of Psychology for Forensic Practitioners. New York: Routledge. 
Warne, T., \& McAndrew, S. (2007). Passive patient or engaged expert? Using a Ptolemaic approach to enhance mental health nurse education and practice. International Journal of Mental Health Nursing, 16, 224-229.

Welch, M. (2005). Pivotal moments in the therapeutic relationship. International Journal of Mental Health Nursing, 14, 161-165. doi:10.1111/j.1440-0979.2005.00376.x.

Wright, S., Sayer, J., Parr, A. M., Gray, R., Southern, D., \& Gournay, K. (2005). Breakaway and physical restraint techniques in acute psychiatric nursing: Results from a national survey of training and practice. The Journal of Forensic Psychiatry and Psychology, 16, 380-398. doi:10.1080/14789940412331270735.

Wynn, R. (2004). Psychiatric inpatients' experiences with restraint. The Journal of Forensic Psychiatry and Psychology, 15, 124-144. doi:10.1080/14789940410001655187. 